\title{
Erratum: Strong new limits on light dark matter from neutrino experiments [Phys. Rev. D 100, 103011 (2019)]
}

\author{
Christopher V. Cappiello® and John F. Beacom
}

(Q) (Received 10 August 2021; published 8 September 2021)

DOI: 10.1103/PhysRevD.104.069901

Due to a numerical error in our propagation code, the nuclear form factor was not properly accounted for when modeling propagation of dark matter (DM) through the Earth's crust. This same issue also affected the interaction of DM particles with target protons within a detector. That error, and the changes described here, affect only our treatment of DM-nucleon scattering and not DM-electron scattering. The most noticeable effect of fixing these errors is to reduce attenuation. So at large cross sections, our limits extend higher by a factor of up to 3.0. At small cross sections, our limits get weaker, but by a negligible amount. Here we revise Figs. 3-6. Figure 7 is also affected, but as it only shows future projections and the effect of these changes is small, we do not reproduce it.

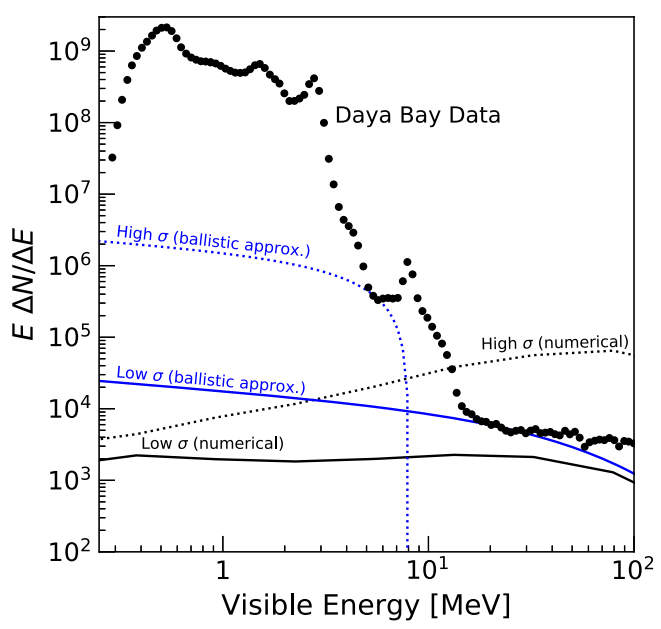

FIG. 1. Updated version of Fig. 3, using the same cross sections as in the main text. The "High $\sigma$ (numerical)" curve is significantly higher than before, while the "Low $\sigma$ (numerical)" curve is slightly lower.

Published by the American Physical Society under the terms of the Creative Commons Attribution 4.0 International license. Further distribution of this work must maintain attribution to the author(s) and the published articles title, journal citation, and DOI. 


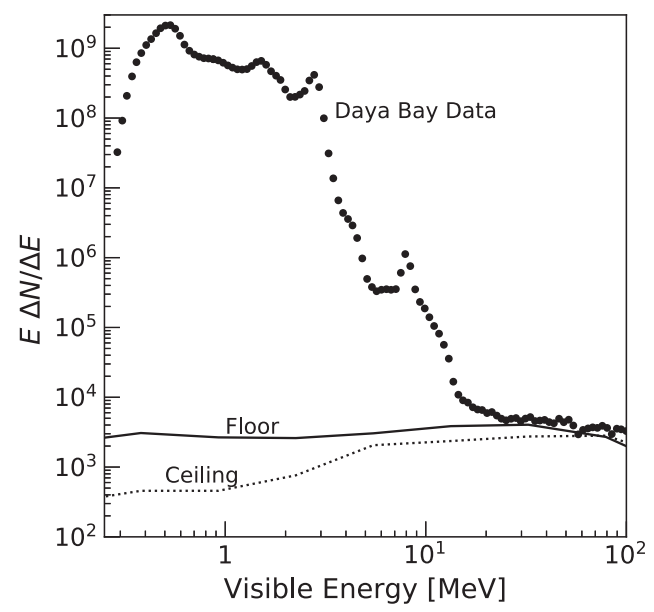

FIG. 2. Updated version of Fig. 4. The "floor" curve now corresponds to $8.0 \times 10^{-29} \mathrm{~cm}^{2}$ instead of $6.0 \times 10^{-29} \mathrm{~cm}^{2}$. The "ceiling" curve now corresponds to $2.3 \times 10^{-27} \mathrm{~cm}^{2}$ instead of $7.0 \times 10^{-28} \mathrm{~cm}^{2}$. These curves are qualitatively similar to the previous curves, because by definition the ceiling and floor are just barely ruled out by the data. This means that fixing the errors in the form factor, and subsequently changing the cross section to correspond to the new floor or ceiling, has the effect of producing similar spectra to those shown previously.

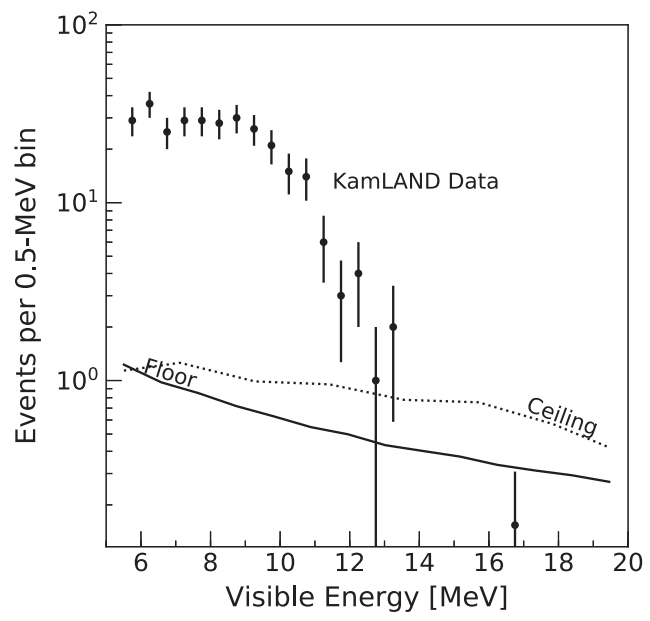

FIG. 3. Updated version of Fig. 5. The "floor" curve now corresponds to $4.0 \times 10^{-31} \mathrm{~cm}^{2}$ instead of $3.0 \times 10^{-31} \mathrm{~cm}^{2}$. The "ceiling" curve now corresponds to $1.5 \times 10^{-28} \mathrm{~cm}^{2}$ instead of $1.3 \times 10^{-28} \mathrm{~cm}^{2}$. These curves are qualitatively similar to the previous curves, for the same reason as above.

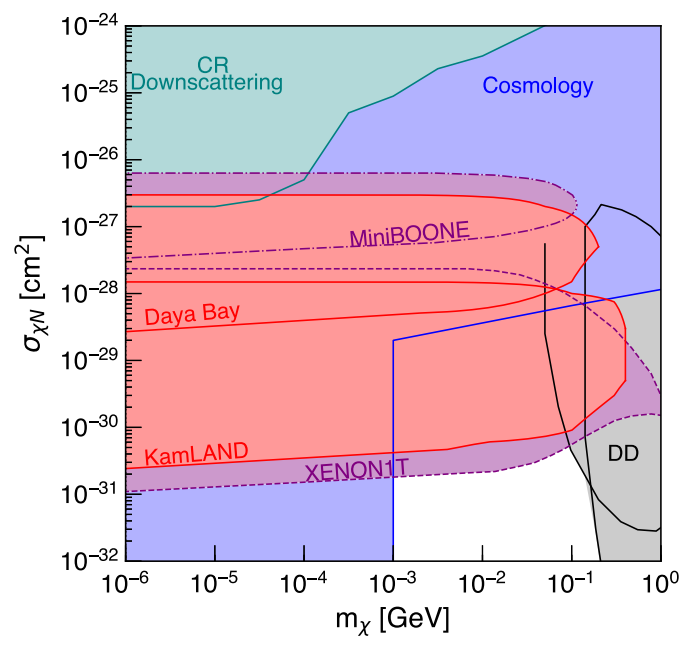

FIG. 4. Updated version of Fig. 6, showing constraints on DM-nucleon scattering. The main change is that the exclusion regions extend to somewhat larger cross sections. The exclusion region for Daya Bay also extends to noticeably larger masses. 


\section{ACKNOWLEDGEMENTS}

We are grateful to Kazumi Hata for pointing out one of these errors. 